\title{
Emerging Information and Communication Technologies for Traffic Estimation and Control
}

\author{
Anastasios Kouvelas $\mathbb{D}^{1},{ }^{1}$ Andy Chow, ${ }^{2}$ Eric Gonzales, ${ }^{3}$ \\ Mehmet Yildirimoglu, ${ }^{4}$ and Rodrigo Castelan Carlson ${ }^{5}$ \\ ${ }^{1}$ École Polytechnique Fédérale de Lausanne, Lausanne, Switzerland \\ ${ }^{2}$ City University of Hong Kong, Kowloon Tong, Hong Kong \\ ${ }^{3}$ University of Massachusetts Amherst, Amherst, MA, USA \\ ${ }^{4}$ University of Queensland, Brisbane, QLD, Australia \\ ${ }^{5}$ Federal University of Santa Catarina, Florianópolis, SC, Brazil \\ Correspondence should be addressed to Anastasios Kouvelas; tasos.kouvelas@epfl.ch
}

Received 25 March 2018; Accepted 26 March 2018; Published 1 August 2018

Copyright (c) 2018 Anastasios Kouvelas et al. This is an open access article distributed under the Creative Commons Attribution License, which permits unrestricted use, distribution, and reproduction in any medium, provided the original work is properly cited.

Information and communication technologies (ICT) are fundamental components of any development in the field of traffic estimation and control. The design and deployment of multimodal advanced transportation systems involve interdisciplinary expertise and effective utilization of new technologies. Nowadays, there are an increasing number of instrumented networks and transportation deployments around the world, and, as a consequence, the research community in this domain has entered the era of big data. The wealthy amount of traffic data that is available in real time can support the development of modern systematic approaches and methodological tools for estimation and control.

Recent advances in transportation (e.g., emerging vehicle communication and automation technologies) have generated new types of measurements, vehicles and infrastructure communications, and control actuation capabilities. All these enable the transportation community to adapt its research topics accordingly and exploit the opportunities resulting from these new developments to improve traffic estimation and control. There is an essential and timely need for new theoretical techniques and algorithms that can accompany the technological achievements and consequently enhance the performance of transportation systems.

The purpose of this special issue is to publish high quality research papers addressing innovative approaches to improve traffic estimation and control by utilizing recent advances and developments in the area of ICT. Among all the submitted manuscripts, six papers were selected for publication on this special issue after a peer review process, as follows.

V. Lücken et al. propose a density-based statistical clustering approach. They present an integrated approach that combines smart street lamps with traffic sensing technology. Infrastructure-based ultrasonic sensors, which are deployed together with a street light system platform, are utilized for multilane traffic participant detection and classification. They develop an algorithmic approach that combines statistical standardization with clustering techniques from the field of unsupervised learning.

$\mathrm{H}$. Chang and B. Yoon proposed a data-driven $k$-nearest neighbour nonparametric regression (KNN-NPR) framework that generates short-term traffic volume predictions. The basic characteristic of their approach is high-speed computational performance when dealing with enormous amounts of historical data. To prove the efficacy of their approach, they have conducted an experimental test with large-size traffic volume data.

Y. Liu and Y. Wang presented and compared the vertical and horizontal queueing modelling paradigms for designing and analysing traffic signal timings in oversaturated conditions with limited mobile sensing data. The analytical results were validated by using AIMSUN model. Their paper 
addressed the temporal consistency between vertical and horizontal queueing models.

R. A. Paielli proposes a standard Trajectory Specification Language (TSL) based on the Extensible Markup Language (XML) for serializing and communicating aircraft trajectories. The language can be used to downlink trajectory requests from air to ground and to uplink trajectory assignments from ground to air. The language is human-readable and may serve as starting point for the development of a communication standard for the Trajectory Specification concept.

X. Hu et al. utilized GPS trajectories from Shenzhen, China, to explore taxi drivers' operation behavior and passengers' demand. Their work focuses on exploring the taxi drivers' operation behavior by utilizing the measurements of activity space and the connection between different activity spaces for different time durations. This research is based on real data and can be helpful for taxi drivers to search for a new passenger and passengers to help them find a taxi's location.

G. A. Klunder et al. presented a study which gives insight into the size of improvement that is possible with individual in-car routing advice based on the actual traffic situation derived from Floating Car Data (FCD). The study uses real loop detector data from the region of Amsterdam, a route generating algorithm for in-car routing advice, and emulated FCD to generate the routing advice. The case with in-car routing advice is compared to the base case, where drivers base their routing decisions on average knowledge of travel times in the network.

Anastasios Kouvelas Andy Chow Eric Gonzales Mehmet Yildirimoglu Rodrigo Castelan Carlson 


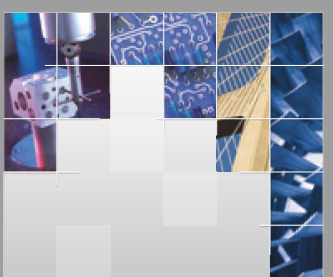

\section{Enfincering}
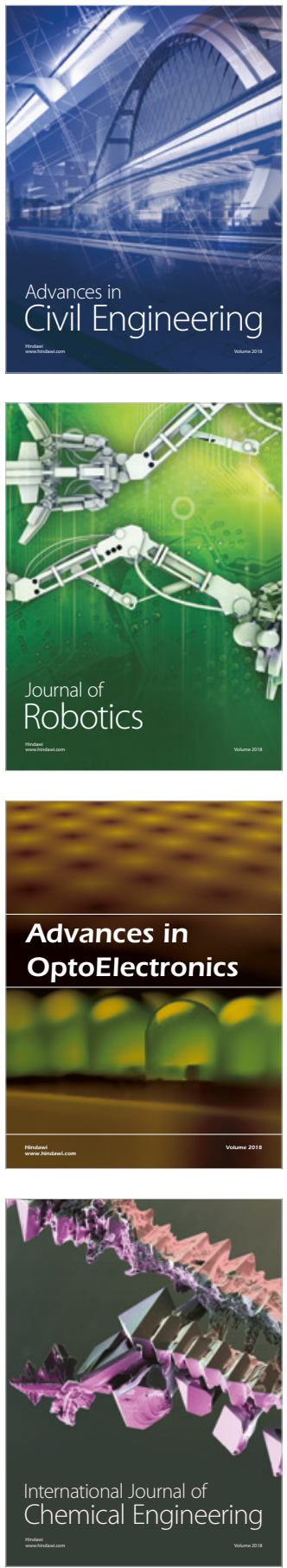

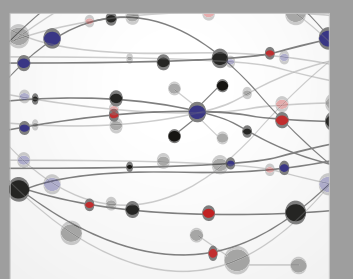

\section{Rotating \\ Machinery}

The Scientific World Journal

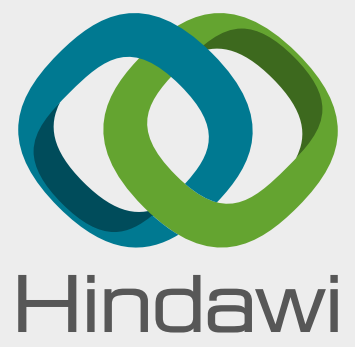

Submit your manuscripts at

www.hindawi.com
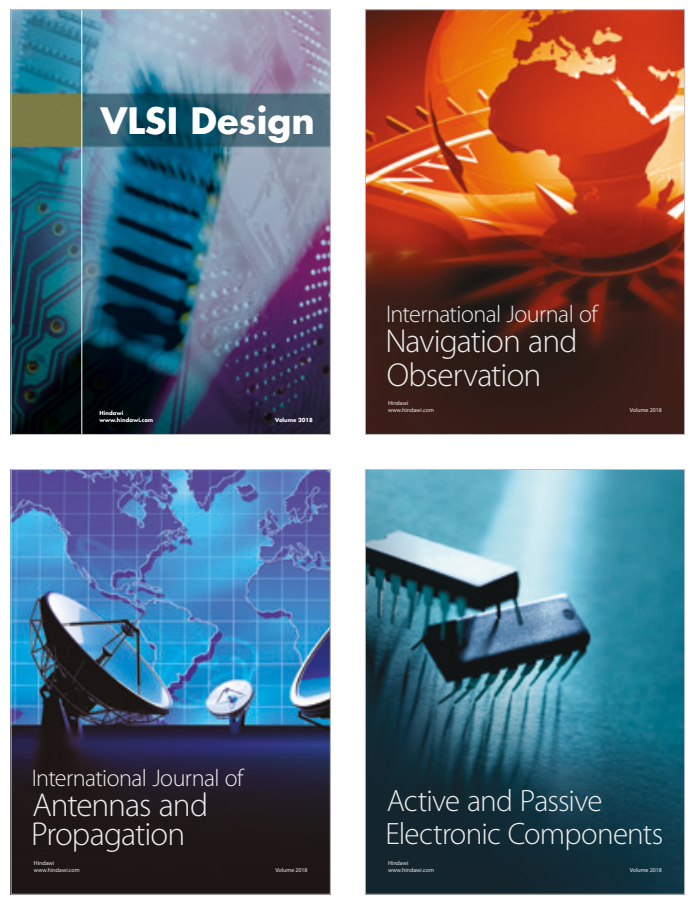
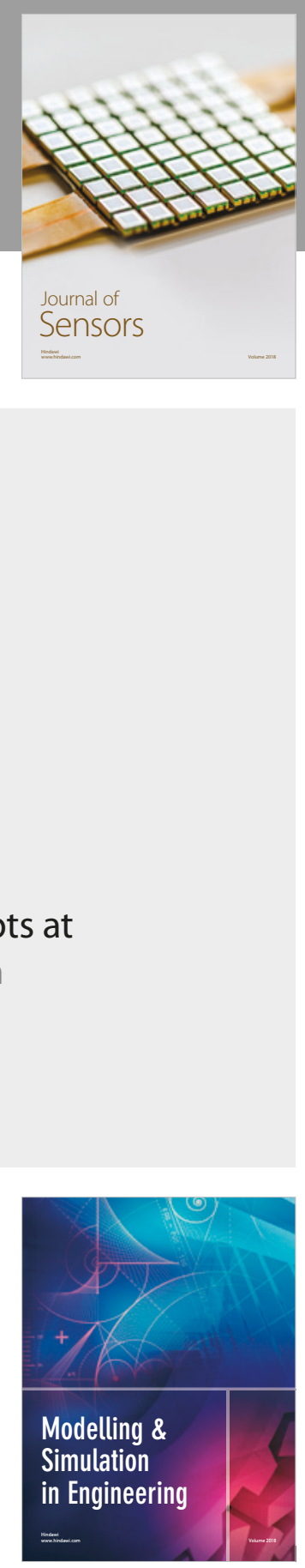

\section{Advances \\ Multimedia}
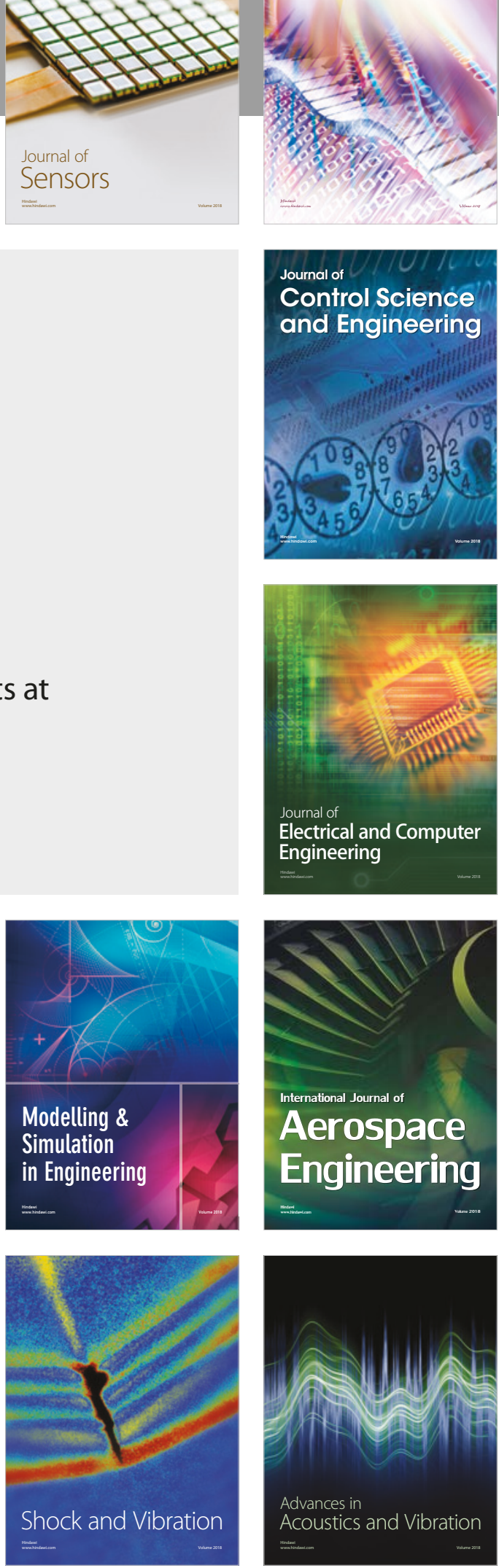